\title{
Irreducible Anterior Shoulder Dislocation Associated With Displaced Fracture of the Greater Tuberosity: An Analysis of Seven Cases
}

\author{
Morteza Nakhaei Amroodii," \\ ${ }^{1}$ Bone and Joint Reconstruction Research Center, Shafa Orthopedic Hospital, Iran University of Medical Sciences, Tehran, IR Iran \\ *Corresponding author: Morteza Nakhaei Amroodi, Bone and Joint Reconstruction Research Center, Shafa Orthopedic Hospital, Iran University of Medical Sciences, Tehran, IR Iran. \\ Tel: +98-9123267519, Fax:+98-2133542020, E-mail: drmna@yahoo.com
}

Received 2015 September 26; Revised 2015 November 4; Accepted 2015 November 13.

\begin{abstract}
Background: Although anterior shoulder dislocation is the most prevalent type of body dislocation, irreducible anterior shoulder dislocation is seldom reported in the literature, which is usually due to physical obstacles.

Objectives: This study presents our findings regarding the causes of irreducibility of anterior shoulder dislocation associated with displaced fracture of the greater tuberosity.

Patients and Methods: CT scans, open reduction of the joint, and internal fixation of the tuberosity was performed in seven patients with irreducible anterior shoulder dislocation associated with displaced fracture of the greater tuberosity.

Results: As confirmed by intraoperative findings, the CT scans showed the cause of irreducible shoulder dislocation in six cases was the interposition of the long head of biceps (LHB) in the anterior of the head that was displaced from the fracture line between the greater and lesser tuberosities. In another case, the greater and lesser tuberosities were attached to each other and were separated from the head. This fractured part was trapped.

Conclusions: We suggest that performing CT scans in all cases of anterior shoulder dislocations with displaced fracture of the greater tuberosity can help surgeons to diagnose the accompanying fractures and possible complications, such as irreducibility. If the fracture line passes through the bicipital groove or in the case of a shield fracture, possible irreducibility should be borne in mind.

Keywords: Computed Tomography, Reduction, Shoulder Fractures, Dislocations
\end{abstract}

\section{Background}

The most common dislocation of the major joints in the human body is that of the glenohumeral joint of the shoulder, and over $95 \%$ of dislocations are anterior dislocations (1). The closed reduction of the anterior dislocated joint is performed under general anesthesia with adequate muscle relaxation accomplished using various maneuvers and techniques (2). Several cases of anterior shoulder dislocations recur after reduction, and various methods have been introduced to decrease the recurrence rate $(3,4)$.

However, there are some cases that do not respond to the initial closed reduction. These dislocations are known as irreducible anterior shoulder dislocations and require open surgery; unfortunately, these are rarely reported on (5). Although arthroscopic intervention has been recently introduced, open surgery still remains the gold standard (1).

As reported in a few cases, some obstacles may interfere with closed reduction, including an interposed bicipital tendon (6), a subscapularis tendon (7), dislocated fragments of the anterior glenoid (8), and greater tuberosity (9).

Preoperative evaluations with computed tomography (CT) scans help physician detect these associated prob- lems and help the surgeon in the operation (10). Yet, there is no consensus regarding the gold standard imaging method for diagnosis, the most suitable surgical approach, postoperative management, or probable complications.

Irreducible anterior shoulder dislocation with fracture of the greater tuberosity has rarely been reported $(9,11)$.

\section{Objectives}

We reviewed our findings of the causes of irreducibility of anterior shoulder dislocation associated with displaced fracture of the greater tuberosity treated 5 to 10 days after the initial injury. The findings were compared with other similar studies.

\section{Patients and Methods}

\subsection{Patients}

In this study, seven patients with irreducible anterior shoulder dislocation associated with displaced fracture of the greater tuberosity were evaluated. All had an un-

Copyright ( ) 2015, Iran University of Medical Sciences. This is an open-access article distributed under the terms of the Creative Commons Attribution-NonCommercial 4.0 International License (http://creativecommons.org/licenses/by-nc/4.0/) which permits copy and redistribute the material just in noncommercial usages, provided the original work is properly cited. 
successful closed reduction under adequate general anesthesia with muscle relaxation and were referred to our center. Patients with irreducible shoulder dislocation but without a greater tuberosity fracture were excluded. This retrospective study was approved by our institutional review board.

\subsection{Preoperative Evaluation}

A physical examination that included the head, neck, chest, abdomen, pelvis, and limbs, especially the injured limb and its neurovascular status, was performed and the results recorded. Anteroposterior and true lateral scapular view X-rays and a three-dimensional CT scan of the affected shoulder were obtained to determine the cause of irreducibility and for operative planning.

\subsection{Operative Technique}

After general anesthesia, the patient was positioned in the beach chair position. The shoulder was explored using the deltopectoral approach; the cephalic vein and deltoid muscle were retracted to the lateral and pectoralis major muscle, and conjugated tendons were retracted to Medial side. The long head of biceps tendon (LHB) was exposed, and the cause of the unsuccessful closed reduction was assessed. The LHB was lacerated in all cases; thus, a tenotomy was done from the supraglenoid tubercle. In cases 1 to 6 , the head was easily reduced after the LHB tenotomy and then the greater tuberosity was fixed transosseously to its anatomic site using heavy non-absorbable sutures. The LHB was then tenodesed to soft tissues around the bicipital groove. In case 7, the LHB tenotomy (from the supraglenoid tubercle) was first performed, but the head could not be reduced. Next, the subscapularis tendon and the infraspinatus tendon were held by stay sutures without being separated from the fractured part and were retracted anteriorly away from the glenoid. The head was then reduced. The fractured part was fixed in the same way, and the LHB was tenodesed.

After confirmation of anatomic reduction with intraoperative Anteroposterior (AP) radiographs, the wounds were closed layer by layer on a hemovaccum drains and dressed. The affected upper limbs were immobilized to the chest by slings and swathes. Two weeks postoperatively, the sutures were removed and intermittent pendulum and passive forward flexion exercises were started. Slings and swathes were removed at four weeks postoperatively, and passive and active assisted exercises for abduction, external rotation, and internal rotation were added gradually to obtain full ranges of motion. Strengthening exercises were started eight weeks postoperatively.

\subsection{Assessments}

A thorough physical examination was performed to rule out possible injuries to other organs, and the involved upper limbs were examined for any injuries to neurovascular structures. Patients' data in terms of age, interval between the accident until closed reduction (hours), interval between the accident until open reduction (days), and the affected shoulder and accident type were recorded. Radiographic studies of the involved shoulders included anteroposterior and true lateral scapular view X-rays. A three-dimensional CT scan was obtained to clarify the cause of irreducibility and for better operative planning.

Intraoperative findings, including the cause of irreducibility, were recorded and confirmation of anatomic reduction was obtained with intraoperative AP radiographs.

\subsection{Statistical Analysis}

There were seven patients with irreducible anterior shoulder dislocation associated with displaced fracture of the greater tuberosity. Even though we gathered all the preoperative and intraoperative findings regarding the cause of irreducibility for the sake of comparison, we found no similar studies except some rare case reports. Therefore, we performed a statistical analysis of some demographic data only.

\section{Results}

Between May 2008 and April 2015, we evaluated and treated seven patients who met the inclusion criteria of this study. All patients had shoulder dislocation with displaced fracture of the greater tuberosity.

Upon a physical examination, anterior shoulder dislocation was detected. All patients were otherwise healthy without any underlying disease, such as coagulopathy, heart, kidney, or liver dysfunction, or history of a previous fracture or upper limb surgery.

All seven patients were male, and the mean age was 30.1 (range: 23 - 42). The mean interval between the accidents and the closed reductions was 8.4 hours, and the mean interval between the accidents and the open reductions was 7 days. Radiographic imaging (anteroposterior and true lateral scapular views) was performed for all cases before the closed reductions, which showed anterior shoulder dislocation with displaced fracture of greater tuberosity in all cases (Figure 1). Five patients had right shoulder dislocations and two had left shoulder dislocations. All were right-handed (Table 1).

Intraoperatively, the cause of the irreducible shoulder dislocation in cases 1 to 6 was found to be the interposition of the LHB in the anterior of the head, which was displaced from the fracture line between the greater and lesser tuberosities into the glenoid and was trapped in front of the head (Figure 2). This type of fracture was obvious on the CT scan and was confirmed during the operation. However, in case 7, the greater and lesser tuberosities were attached to each other and both were separated from the head, which is called a "shield fracture". This fractured part was trapped in front of the head, be- 
cause it was attached to the subscapularis tendon from the medial side and to the infraspinatus tendon from the lateral side. These muscles pushed the head in anterior of glenoid to medial side strongly and prohibited depart- ing the head into the glenoid (Figure 2). The LHB was lacerated in all cases and therefore was tenotomized from the supraglenoid tubercle and tenodesed to soft tissues around the bicipital groove.

Figure 1. Radiographic Results of Cases (all Similar)
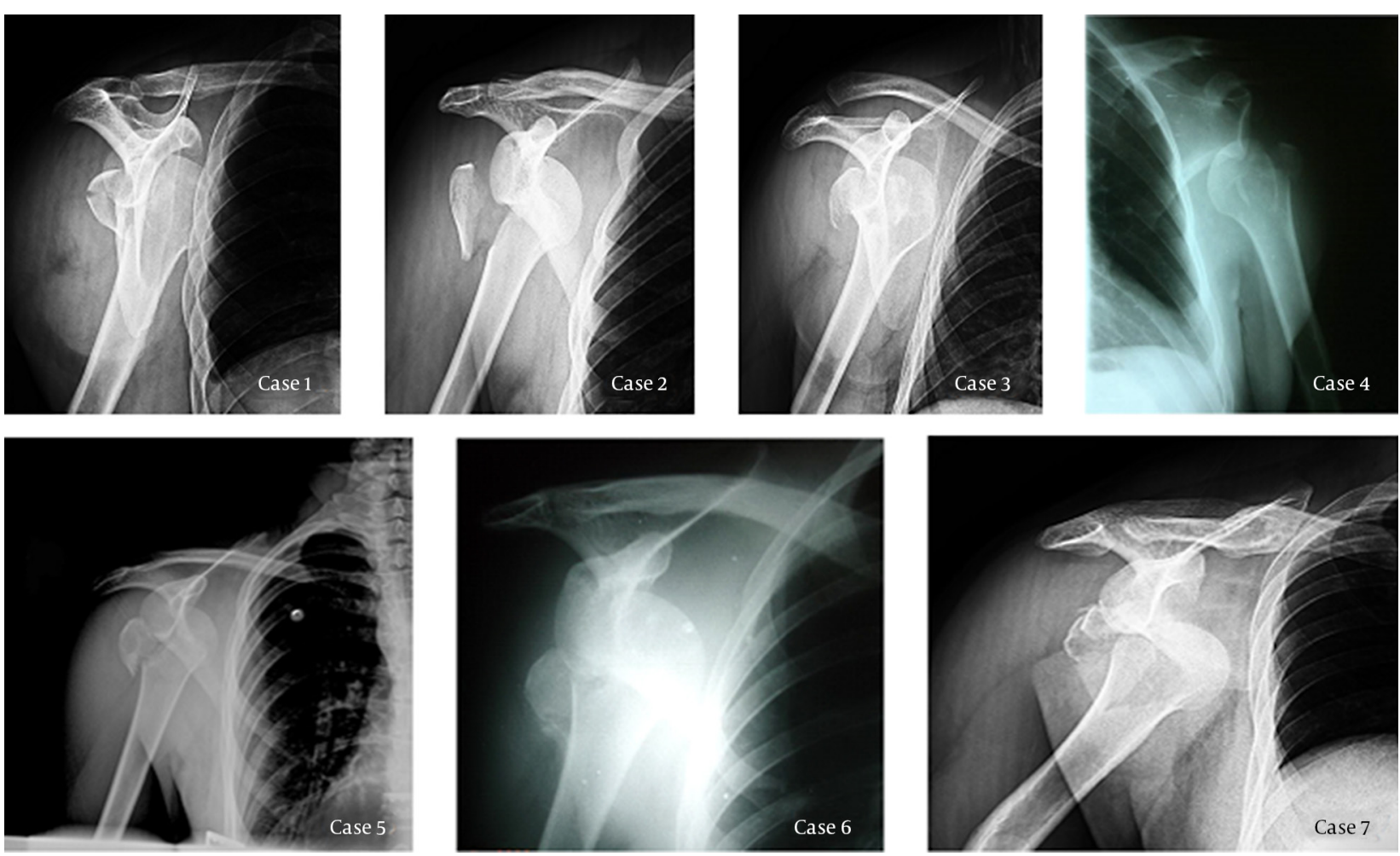

Anterior shoulder dislocation with displaced fracture of greater tuberosity.

Table 1. Detailed Information About Patients

\begin{tabular}{|c|c|c|c|c|c|c|}
\hline Case No. & Age, y & $\begin{array}{l}\text { Interval Between the Accident } \\
\text { and Closed Reduction, } h\end{array}$ & $\begin{array}{l}\text { Interval Between } \\
\text { the Accident and } \\
\text { Open Reduction, d }\end{array}$ & $\begin{array}{l}\text { Involved } \\
\text { Shoulder }\end{array}$ & Dexterity & Accident Type \\
\hline $\mathbf{1}$ & 42 & 10 & 8 & Right & Right & Pedestrian hit by car \\
\hline 2 & 38 & 8 & 7 & Right & Right & Motorcycle accident \\
\hline 3 & 23 & 6 & 10 & Right & Right & Falling from height \\
\hline 4 & 23 & 9 & 6 & Left & Right & Motorcycle accident \\
\hline 5 & 28 & 11 & 7 & Right & Right & Pedestrian hit by car \\
\hline 6 & 33 & 8 & 5 & Left & Right & Motorcycle accident \\
\hline 7 & 24 & 7 & 6 & Right & Right & Car accident \\
\hline
\end{tabular}


Figure 2. CT Scan Results of Cases
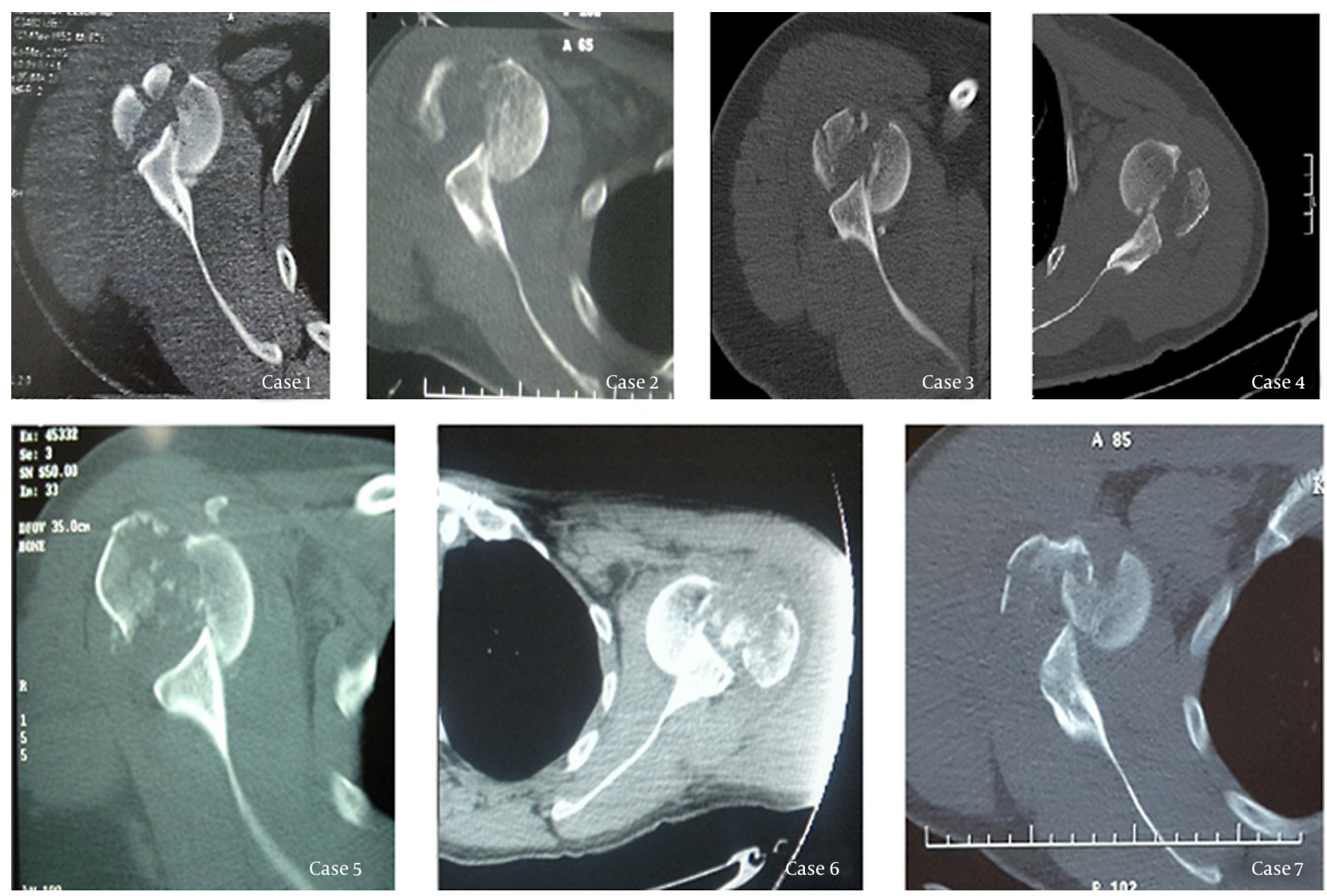

Anterior shoulder dislocation with displaced fracture of greater tuberosity. The fracture line crossed the bicipital groove in cases 1 to 6 and presence a shield fracture in case 7.

\section{Discussion}

As the findings of this study indicated, all patients had similar radiographic imaging results (Figure 1), but case 7 had a shield fracture that was only evident upon the CT scan (Figure 2 ) and that required additional intervention in the surgical procedure.

Allard et al. demonstrated in one case of irreducible anterior shoulder dislocation that simple radiography might not be enough to properly assess shoulder dislocation and that a CT scan and magnetic resonance imaging (MRI) are necessary (10). In their study, MRI was required in addition to a CT scan to obtain a complete picture. However, our study demonstrated the adequacy of a CT scan in assessing patients with anterior shoulder dislocation with a greater tuberosity fracture. Bridle et al. have suggested an additional radiographic image in old patients and those with significant pain (12), which demonstrates the importance of sufficient imaging before intervention.

There are few reported cases of irreducible anterior shoulder dislocation with a fracture of the greater tuberosity. Aiyenuro and Zadeh reported a case with a combination of a greater tuberosity fracture, a rotator cuff tear, and a supra- spinatus avulsion with a Hill-Sachs lesion repaired using the McKenzie approach. They concluded that this method made exposure and repair easy (13). Although their case was more complicated than our cases, our results showed there was no difficulty performing the procedures using the deltopectoral approach. Ilahi also, reported a case of irreducible anterior shoulder dislocation with a fracture of the greater tuberosity who required open reduction and division of the subscapularis tendon before reduction of the glenohumeral dislocation (11).

Other studies have reported cases with irreducible anterior shoulder dislocation due to other mechanisms, such as an interposed bicipital tendon (6), a subscapularis tendon $(7,12)$, and dislocated fragments of the anterior glenoid (8). These are all uncommon and require surgical treatment.

Various studies have reported late presentation or very muscular individuals as reasons for the failure of reduction (14-16). The mean time of interval between accident and closed reduction ranged from 6 to 11 hours, which indicates that all cases were referred in time for closed reduction and that the cause of irreducibility was related to a fracture of the greater tuberosity. 
Bahrs et al. investigated the morphology and mechanisms of greater tuberosity fractures and found that 59/103 (57.2\%) were associated with shoulder dislocations (17). Therefore, paying attention to all accompanying fractures is necessary in the management of shoulder dislocations. This can be accomplished using detailed imaging methods, such as CT scans.

As stated, there are very few reported cases of irreducible anterior shoulder dislocation. Thus, we aimed to report the results of seven cases to help improve understanding. Yet, more detailed studies and review studies are needed to give physicians and surgeons a better understanding of the most appropriate diagnostic tools and surgical approaches.

Although irreducible anterior shoulder dislocation is not very common, it is very important to inform the patient that their dislocation might be associated with complications, such as irreducibility, and may not be easily treated by closed reduction but may require surgical intervention. The necessary equipment and skilled surgeons must be available to manage the patient properly.

The experience of our seven cases with irreducible anterior shoulder dislocation with a displaced fracture of the greater tuberosity revealed that performing CT scans can help the surgeon to diagnose the accompanying fractures and possible complications to be prepared for other potential interventions. These dislocations may be irreducible if the greater tuberosity was detached from the bicipital groove or if a shield fracture was present anterior to the head.

\section{References}

1. Cutts S, Prempeh M, Drew S. Anterior shoulder dislocation. Ann R Coll Surg Engl. 2009;91(1):2-7. doi: 10.1308/003588409X359123. [PubMed:19126329]

2. Kuhn JE. Treating the initial anterior shoulder dislocation-an evidence-based medicine approach. Sports Med Arthrosc. 2006;14(4):192-8. doi: 10.1097/01.jsa.0000212328.25345.de. [PubMed:17135968]

3. Itoi E, Hatakeyama Y, Kido T, Sato T, Minagawa H, Wakabayashi I, et al. A new method of immobilization after traumatic anterior dislocation of the shoulder: a preliminary study.J Shoulder Elbow Surg. 2003;12(5):413-5. doi: 10.1016/S105827460300171X. [PubMed: 14564258]

4. Itoi E, Hatakeyama Y, Sato T, Kido T, Minagawa H, Yamamoto N, et al. Immobilization in external rotation after shoulder dislocation reduces the risk of recurrence. A randomized controlled trial. J Bone Joint Surg Am. 2007;89(10):2124-31. doi: 10.2106 JBJS.F.00654. [PubMed:17908886]

5. Guha AR, Jago ER. Irreducible acute anterior shoulder dislocation. Int J Clin Pract. 2004;58(12):1184-6. [PubMed:15646422]

6. Day MS, Epstein DM, Young BH, Jazrawi LM. Irreducible anterior and posterior dislocation of the shoulder due to incarceration of the biceps tendon. Int J Shoulder Surg. 2010;4(3):83-5. doi: 10.4103/0973-6042.76970. [PubMed: 21472069]

7. Connolly S, Ritchie D, Sinopidis C, Brownson P, Aniq H. Irreducible anterior dislocation of the shoulder due to soft tissue interposition of subscapularis tendon. Skeletal Radiol. 2008;37(1):63-5. doi:10.1007/s00256-007-0370-y. [PubMed:17912520]

8. Mihata T, Doi M, Abe M. Irreducible acute anterior dislocation of the shoulder caused by interposed fragment of the anterior glenoid rim. J Orthop Sci. 2000;5(4):404-6. doi: 10.1007| s007760000050404.776. [PubMed:10982692]

9. Oni OO. Irreducible acute anterior dislocation of the shoulder due to a loose fragment from an associated fracture of the greater tuberosity. Injury. 1983;15(2):138. [PubMed: 6629490]

10. Allard JC, Bancroft J. Irreducible posterior dislocation of the shoulder: MR and CT findings. J Comput Assist Tomogr. 1991;15(4):694-6. [PubMed: 2061493]

11. Ilahi OA. Irreducible anterior shoulder dislocation with fracture of the greater tuberosity. Am J Orthop (Belle Mead NJ). 1998;27(8):576-8. [PubMed: 9732082]

12. Bridle SH, Ferris BD. Irreducible acute anterior dislocation of the shoulder: interposed scapularis. J Bone Joint Surg Br. 1990;72(6):1078-9. [PubMed: 2246295]

13. Aiyenuro OD, Zadeh HG. Irreducible acute anterior shoulder dislocation due to rotator cuff tear and associated fracture of the greater tuberosity, A method of open reduction and rotator cuff repair. Injury Extra. 2007;38(3):74-7. doi: 10.1016/j.injury.2006.07.050.

14. Gleeson AP. Anterior glenohumeral dislocations: what to do and how to do it.J Accid Emerg Med.1998;15(1):7-12. [PubMed: 9475214]

15. Schulz TJ, Jacobs B, Patterson RL. Unrecognized dislocations of the shoulder.J Trauma. 1969;9(12):1009-23. [PubMed: 5358646]

16. McMahon PJ, Lee TQ. Muscles may contribute to shoulder dislocation and stability. Clin Orthop Relat Res. 2002;(403 Suppl):S18-25. [PubMed: 12394449]

17. Bahrs C, Lingenfelter E, Fischer F, Walters EM, Schnabel M Mechanism of injury and morphology of the greater tuberosity fracture. J Shoulder Elbow Surg. 2006;15(2):140-7. doi: 10.1016/j. jse.2005.07.004. [PubMed:16517355] 